[5] Gabbay S, McQueen DM, Yellin EL, Becker RM, Frater RW. In vitro hydrodynamic comparison of mitral valve prostheses at high flow rates. J Thorac Cardiovasc Surg 1978;76(6):771-87.

[6] Saikrishnan N, Yap CH, Milligan NC, Vasilyev NV, Yoganathan AP. In vitro characterization of bicuspid aortic valve hemodynamics using particle image velocimetry. Ann Biomed Eng 2012;40(8):1760-75.

[7] Casale PN, Palacios IF, Abascal VM, et al. Effects of dobutamine on Gorlin and continuity equation valve areas and valve resistance in valvular aortic stenosis. Am J Cardiol 1992;70(13):1175-9.
[8] Gilon D, Cape EG, Handschumacher MD, et al. Effect of three-dimensional valve shape on the hemodynamics of aortic stenosis: three-dimensional echocardiographic stereolithography and patient studies. J Am Coll Cardiol 2002;40(8):1479-86.

[9] Clark C. The fluid mechanics of aortic stenosis-I. Theory and steady flow experiments. J Biomech 1976;9(8):521-8.

[10] Bermejo J. Estimation of the end of ejection in aortic stenosis: an unreported source of error in the invasive assessment of severity. Circulation 2004;110(9):1114-20.

\title{
Acute effect of isometric resistance exercise on blood pressure of normotensive healthy subjects
}

\author{
Carlos Alberto da Silva ${ }^{\mathrm{a}, *}$, Arnaldo Mortatti ${ }^{\mathrm{a}}$, Ricardo Pereira Silva ${ }^{\mathrm{b}}$, Geraldo B. Silva Jr. ${ }^{\text {c,d }}$, \\ Victor Fernando Tavares Erberelli ${ }^{\mathrm{e}}$, Fabiano Stefanini ${ }^{\mathrm{e}}$, Marcos Rocha Lima ${ }^{\mathrm{e}}$ \\ a Institute of Physical Education and Sports, Federal University of Ceará, Fortaleza, Ceará, Brazil

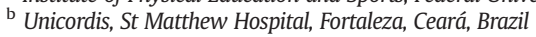 \\ c Department of Internal Medicine, School of Medicine, Federal University of Ceará, Fortaleza, Ceará, Brazil \\ d School of Medicine, Health Sciences Center, University of Fortaleza, Fortaleza, Ceará, Brazil \\ e Stúdio Master Fitness Academy, Fortaleza, Ceará, Brazil
}

\section{A R T I C L E I N F O}

\section{Article history:}

Received 7 January 2013

Accepted 29 March 2013

Available online 2 May 2013

\section{Keywords:}

Isometric resistance exercise

Cardiovascular risk

Blood pressure

Heart rate

Double product

Cardiovascular diseases (CVD) are the main cause of morbidity and mortality in the general population [1]. Hypertension is a well established risk factor for the development of CVD, and its incidence increases with age [2]. Resistance exercise, dynamic [3,4] or isometric [5-7] have been used in the treatment of hypertension. However it is still not clear which is the impact of a session of isometric resistance exercise (IRE) in blood pressure (BP), i.e., its acute effect.

According to Hagberg et al. [8], there is concern regarding that this type of exercise increases $\mathrm{BP}$ and is not a conditioning exercise, like the dynamic exercise, which is a conditioning exercise, and lowers resting BP and heart rate (HR). O'Hare and Murnaghan [9] pointed the risks of IRE in individuals with compromised cardiovascular function, including elderly people, or potentially compromised, such as left ventricule dysfunction, CVD, cardiomyopathy, aortic dilation and others, although IRE cronically decreases BP [10].

This spectrum becomes even more important because there has been a craze in which body fitness and isometric exercises are performed daily by many people in fitness centers or at home regardless of BP levels [11].

\footnotetext{
* Corresponding author at: Instituto de Educação Física e Esportes, Universidade Federal do Ceará, Campus do Pici, Bloco 320, Av. Mister Hull, S/N. CEP 60455-760, Fortaleza, Ceará, Brazil. Tel.: +55 8533669533.

E-mail addresses: carlosas@ymail.com (C.A. da Silva), geraldobezerrajr@yahoo.com.br (G.B. Silva).
}

A total of 24 normotensive healthy individuals, males, aged 2040 years, physically active were included. The study was conducted at the Health and Physical Activity Laboratory, Physical Exercises and Sports Institute, Federal University of Ceará, Fortaleza, Ceará, Brazil. All participants underwent an IRE session. One day before the exercise, a maximum voluntary contraction (MVC) test was done, with 10 maximal repetitions (RM) [12]. After MCV test they were randomized in three groups: (LI) low intensity and low duration, characterized as low intensity; (MI) high intensity and low duration, characterized as moderate intensity; and (HI) low intensity and high duration, characterized as high intensity. In both programs the participants performed exercise one time. Each group performed IRE protocol: LI (20\% intensity, 3 series, duration $30 \mathrm{~s}$ for each sets) (sets composition with three muscular actions of $10 \mathrm{~s}$ of three different angle contractions), 1 min of interval between the series, exercise Leg Press Inclinade $\left(45^{\circ}\right)$; MI (20\% intensity, 3 series, duration 1 min each series) (series composition with one muscular action, 1 min of angle contraction), 1 min of interval between the series, exercise Leg Press Inclinade $\left(45^{\circ}\right)$; HI $(60 \%$ intensity, 3 series, $30 \mathrm{~s}$ duration for each series) (series composition with three muscular actions of $10 \mathrm{~s}$ of contraction in three different angles), $1 \mathrm{~min}$ of interval between the series, exercise Leg Press Inclinade $\left(45^{\circ}\right)$. All participants underwent a MCV test, which is the maximum number of repetitions by series that can be done with the correct technique, with a given charge. The higher charge that can be used in an exercise repetition is considered 1-RM.

Table 1

Baseline characteristics of the subjects in the study, in their respective groups.

\begin{tabular}{lcccl}
\hline Characteristics & HI $(\mathrm{n}=8)$ & MI $(\mathrm{n}=8)$ & LI $(\mathrm{n}=8)$ & $\mathrm{p}$ \\
\hline Age $($ years $)$ & $30.6 \pm 6.2$ & $31.6 \pm 6.6$ & $27.5 \pm 4.6$ & 0.35 \\
Body mass $(\mathrm{kg})$ & $74.4 \pm 8.6$ & $72.3 \pm 13.9$ & $74.2 \pm 15.8$ & 0.93 \\
Height $(\mathrm{m})$ & $174.4 \pm 3.9$ & $171.8 \pm 9.1$ & $169.6 \pm 8.5$ & 0.46 \\
BMI $\left(\mathrm{kg} / \mathrm{m}^{2}\right)$ & $24.7 \pm 2.6$ & $24.2 \pm 2.7$ & $25.5 \pm 3.1$ & 0.64 \\
HR $($ beats $/ \mathrm{min})$ & $78.4 \pm 11.0$ & $88.4 \pm 13.8$ & $83.3 \pm 15.0$ & 0.34 \\
MBP $(\mathrm{mm} \mathrm{Hg})$ & $89.6 \pm 12.8$ & $93.9 \pm 11.4$ & $81.9 \pm 9.8$ & 0.39 \\
MVC $(\mathrm{kg})$ & $253.9 \pm 68.8$ & $273.7 \pm 56.2$ & $224.6 \pm 64.9$ & 0.31 \\
\hline
\end{tabular}

Note: values are means \pm SD

Abbreviations: BMI: body mass index. HR: heart rate. MBP: medium blood pressure. MVC: maximal voluntary contraction. 




Fig. 1. Graphic representation of the heart rate values as a function of isometric exercise in groups during the sets. ${ }^{*}$ Significant difference between 1 set to 3 sets in HI group $(\mathrm{p}<0.01)$.

As shown in Table 1, the three groups presented similar characteristics. There was no significant difference between the groups regarding age, MBP, MVC and height (Table 1). In all the groups there was a significant increase in HR in all the series regarding the interval period. Fig. 1 presents the results verified post hoc in the evolution of IRE execution in the three protocols. It was observed that only the HI protocol had an influence in the number of series and/or work charge, generating a cardiovascular response with very elevated magnitude $(\mathrm{p}<0.01)$, which did not occur in the groups LI and MI. The cumulative effect of the series seems to generate fatigue, increasing HR, probably to compensate the exercised muscle. This seems to occur more frequently according to the muscle size (the larger the exercised muscle the higher the effects on HR). The results of BP as a result of IRE session showed more sensitive, as showed in Table 2 and Fig. 2. In SBP there was a significant difference in LI group compared to $\mathrm{HI}$ ( 1 set of LI to 3 sets of HI $\mathrm{p}<0.001$ ), but not between LI and MI, showing that IRE training with high intensity increases the cardiovascular risk. In DBP there was a significant difference in group HI ( 1 set of HI to 3 sets of $\mathrm{HI}, \mathrm{p}<0.04$ ) and between the groups, indicating that in groups MI and HI the individuals are exposed to a higher cardiovascular risk ( 1 set of LI to 3 sets of MI, $\mathrm{p}<0.04$, and 1 set of LI to 3 sets of $\mathrm{HI}, \mathrm{p}<0.003$ ). In MBP, there was a significant difference to groups $\mathrm{MI}$ and $\mathrm{HI}$ compared with $\mathrm{LI}$ ( 1 set of LI to 3 sets of MI, $\mathrm{p}<0.02$, and 1 set of LI to 3 sets of HI, $\mathrm{p}<0.01$ ), as well as in group $\mathrm{HI}$ ( 1 set of $\mathrm{HI}$ to 3 sets of $\mathrm{HI}, \mathrm{p}<0.01$ ), showing a higher risk when practicing IRE with moderate and high intensities (Fig. 3). In DP there was a significant difference in group $\mathrm{HI}$ ( 1 set of $\mathrm{HI}$ to 3 sets of $\mathrm{HI}$, $\mathrm{p}<0.05$ ) and between groups BI and HI ( 1 set of LI to 3 sets of HI, $\mathrm{p}<0.05$ ), showing an important effect similar to BP, evidencing an increased risk of executing IRE with high intensity. The main finding in the present study was that IRE generates an imminent cardiovascular risk during its execution and progressively increases BP if executed with high intensity (charge $<70 \%$ MVC) in healthy normotensive individuals. The cumulative increase in the number of series also seems to increase BP.

This increase in BP during the execution of acute IRE seems to be mainly due to an increase in cardiac output (CO), and not due to increase in peripheral vascular resistance (PVR). These findings generate interesting information, which justifies a better control of the performance of IRE in healthy places, mainly when the individual health status is not known. It is then worth to reinforce that if it is indicated, the execution of IRE should be done with low intensity (charge $\geq 30 \%$ MVC) and fast execution (10 and $15 \mathrm{~s}$ ).

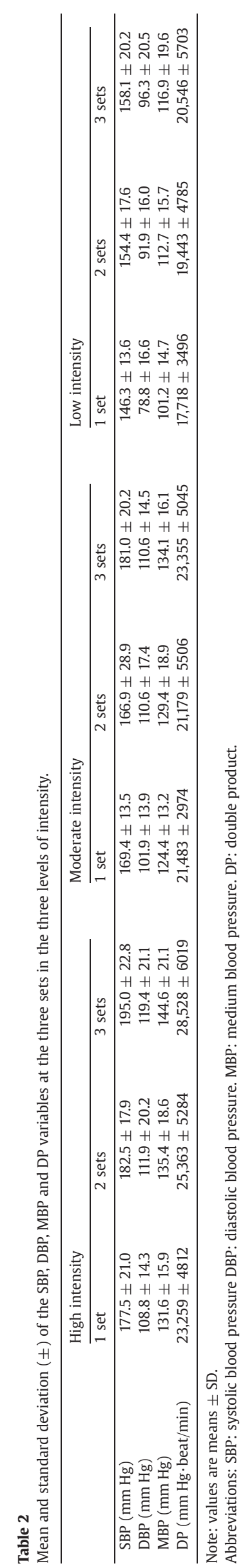



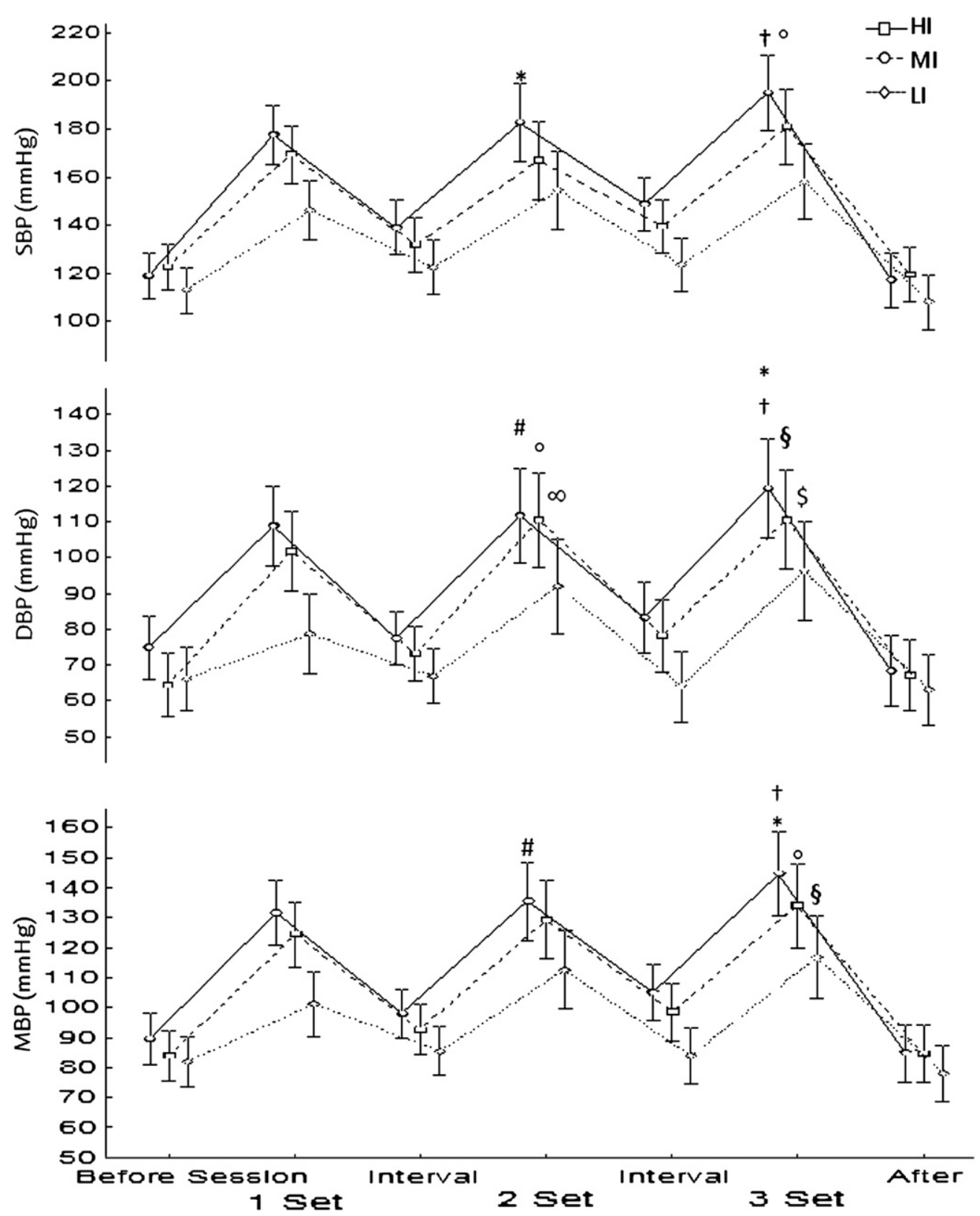

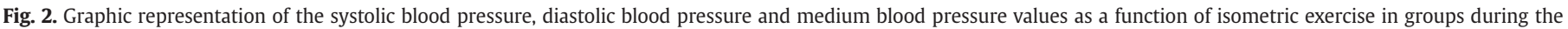

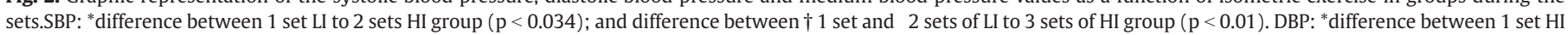

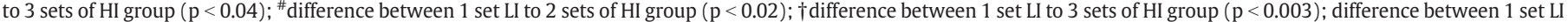

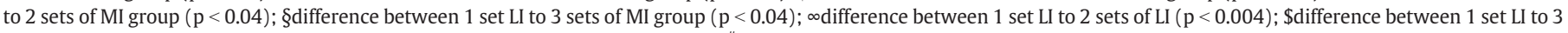

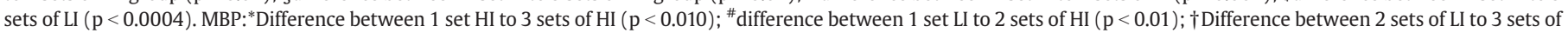
$\mathrm{HI}(\mathrm{p}<0.03)$; difference between 1 set of LI to 3 sets of MI $(\mathrm{p}<0.02)$; §difference between 1 set of LI to 3 sets of LI ( $p<0.003)$.

\section{References}

[1] Roger VL, Go AS, Lloyd-Jones DM, et al. Heart disease and stroke statistics - 2011 update: a report from the American Heart Association. Circulation 2011;123(4):e18

[2] Standridge JB. Hypertension and atherosclerosis: clinical implications from the ALLHAT trial. Curr Atheroscler Rep 2005;7(2):132.

[3] Cbobanian AV, Bakris GL, Black HR, et al. Seventh report of the Joint National Committee on prevention, Detection, Evaluation, and Treatment of High Blood Pressure. Hypertension 2003:42:1206.

[4] Mancia G, Debacker G, Dominiczak A, et al. 2007 Guidelines for the management of arterialhypertension: the Task Force for the Management of Arterial Hypertension of the European Society of Hypertension (ESH) and of the European Society Cardiology (ESC). J Hypertens 2007;25:1105

[5] Felici F, Quarsima V, Fattorini L, Sbriccoli P, Filligoi GC, Ferrari M. Biceps brachii myoeletric and oxygenation changes during static and sinusoidal isometric exercises. Electromyogr Kinesiol 2009;92:e1.
[6] Devereux GR, Wiles JD, Swaine IL. Reductions in resting blood pressure after 4 weeks of isometric exercise training. Eur J Appl Physiol 2010;109:601

[7] Wiles JD, Coleman DA, Swaine IL. The effects of performing isometric training at two exercise intensities in healthy young males. Eur J Appl Physiol 2010;108:419.

[8] Hagberg JM, Park JJ, Brown MD. The role of exercise training in the treatment of hypertension: an update. Sports Med 2000;30:193-206.

[9] O'Hare JA, Murnaghan DJ. Failure of anti-hypertensive drugs to control blood pressure rise with isometric exercise in hypertension. Postgrad Med J 1981;57:552.

[10] Millar PJ, Bray SF, McGowan CL, et al. Effects of isometric handgrip training among people medicated for hypertension: a multilevel analysis. Blood Press Monit 2007; $12: 307$.

[11] Chrysant SG. Current evidence on the hemodynamic and blood pressure effects of isometric exercise in normotensive and hypertension persons. J Clin Hypertens 2010;12:721.

[12] Bryzcki M. Strength test: predicting a one-rep max from a reps-to-fatigue. Journal of Physical Education, recreation and Dance 1993;64:88-90. 


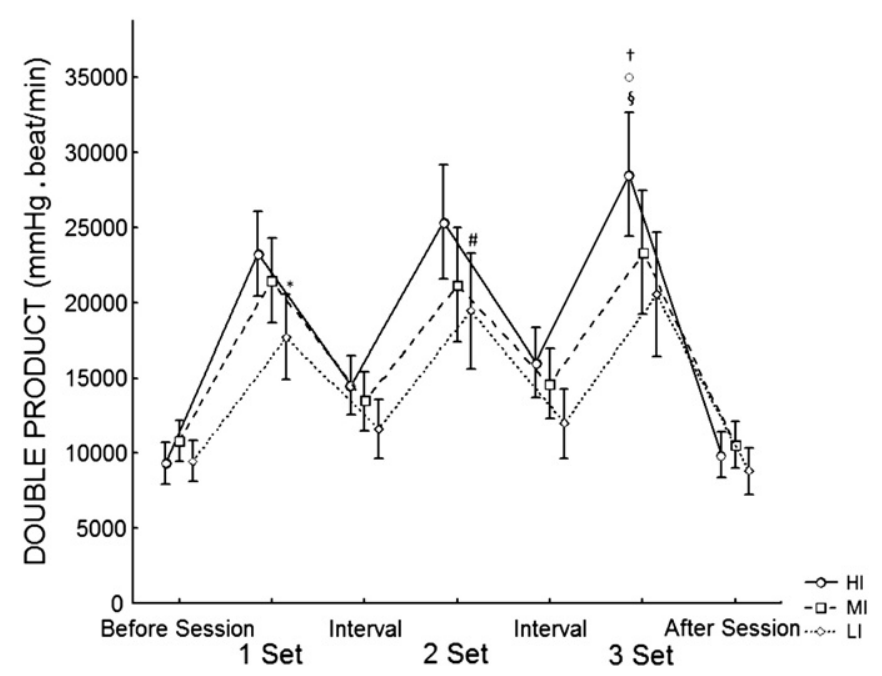

Fig. 3. Graphic representation of the double product (product of blood pressure by heart rate - RPP) values as a function of isometric exercise in groups during the sets. ${ }^{*}$ Difference between 1 set to 3 sets of LI group ( $\left.\mathrm{p}<0.05\right)$; ${ }^{\text {\#Difference between } 2}$ sets of $\mathrm{LI}$ to 1 set of HI group ( $\mathrm{p}<0.01$ ); difference between 3 sets of $\mathrm{HI}$ to $\dagger 1$ set of $\mathrm{HI}$ group, $\S 1$ set of $\mathrm{LI}, 2$ sets of $\mathrm{LI}(\mathrm{p}<0.05)$.

\title{
Prevalence of classical CD14 $++/$ CD16 - but not of intermediate CD14 $++/$ CD16 + monocytes in hypoalphalipoproteinemia
}

\author{
Federica Sala ${ }^{\mathrm{a}, 1}$, Lucia Cutuli a,1, Liliana Grigore ${ }^{\mathrm{b}, \mathrm{c}}$, Angela Pirillo ${ }^{\mathrm{b}}$, Giulia Chiesa ${ }^{\mathrm{a}}$, Alberico Luigi Catapano ${ }^{\mathrm{a}, \mathrm{c}}$, \\ Giuseppe Danilo Norata ${ }^{\mathrm{a}, \mathrm{b}, \mathrm{d}, *}$ \\ a Department of Pharmacological and Biomolecular Sciences, Università degli Studi di Milano, Milan, Italy \\ b Center for the Study of Atherosclerosis, Società Italiana Studio Aterosclerosi, Ospedale Bassini, Cinisello Balsamo, Italy \\ c IRCCS Multimedica, Milan, Italy \\ d The Blizard Institute, Centre for Diabetes, Barts and The London School of Medicine E Dentistry, Queen Mary University, London, UK
}

\section{A R T I C L E I N F O}

\section{Article history:}

Received 31 December 2012

Accepted 29 March 2013

Available online 23 April 2013

\section{Keywords:}

Hypoalphalipoproteinemia monocytes

HDL

Immunity

ApoA-

\section{To the Editor,}

In humans, monocytes display heterogenicity related to the surface expression of the lipopolysaccharide receptor (CD14) and the low affinity FCgamma-receptor-III (CD16) which results in the presence of three subsets: classical (CD14++/CD16-), intermediate $(\mathrm{CD} 14++/ \mathrm{CD} 16+)$

\footnotetext{
* Corresponding author at: Department of Pharmacological and Biomolecular Sciences, University of Milan, Via Balzaretti 9, 20133, Milan, Italy. Tel.: + 3902 50318313; fax: + 39 0250318386.

E-mail address: danilo.norata@unimi.it (G.D. Norata).

1 These authors equally contributed to this work.
}

and non-classical (CD14 +/CD16++) monocytes. The association of the different subsets with cardiovascular disorders is highly debated; classical monocytes CD14++/CD16- predict cardiovascular events in a randomly selected population [1], but a recent report by Rogacev KS et al. [2] showed that CD14++/CD16 + intermediate but not classical monocytes are the best predictor in subjects referred for elective coronary angiography. These contrasting findings might indicate that specific monocyte subsets could be enriched under different inflammatory conditions associated to cardiovascular disorders and prompt the need for a detailed subset analysis in specific CVD contexts.

Among these, low high-density lipoprotein cholesterol (HDL-C) levels are associated to increased inflammation and coronary heart disease (CHD) [3] and therefore we aimed to study the distribution of different monocyte subsets in relation to HDL-C plasma levels particularly in the context of hypoalphalipoproteinemia. First we analyzed the correlation between monocyte subsets and HDL in subjects randomly selected from a population enrolled in a longitudinal observational study (PLIC study [4]; the informed consent was obtained from each patient and the study protocol conforms to the ethical guidelines of the 1975 Declaration of Helsinki as reflected in a priori approval by the institution's human research committee) and observed an inverse correlation between the percentage of classical monocytes and the levels of HDL cholesterol and a direct correlation between the percentage of the "non-classical" monocytes with HDL cholesterol (Fig. 1A and B). No correlation of HDL with the 